Ramsey Rolda R. Una ricerca fotografica di pile elettrolitiche (p. 269). - Vedi N. Cim., serie IV, vol. X, p. 461.

Rood O. N. Sul fotometro a scintillamento (pp. 269-271). Breve sunto di notizie riguardanti i vari perfezionamenti intro. dotti in questu fotometro.

Sнend Joнx C. Studio della radiazione in un campo magne. tico mediante l'interferometro (pp. 270-272). - Vedi N. Cim., serie IV, vol. $X$, pp. 392 e 396.

RIEOKe Edodrd. Sulla variazione e sull' equilibrio di sistemi polari trigonali. Contributo alla teoria della struttura cristallina (pp. 277-282).

MAC GREGor J. G. a Maddonald W. A. Sulla variazione delle proprietà elastiche del czucciù vulconizaato (p. 282). - La rigidita del caucciù $\theta$ il modulo di Joung diminuiscono in certi casi colla trazione fino ad tu minimo, per poi aumentare.

Van Aubel Edmond. Osservazione sulla comunicazione del Sig. Francis Magie \& Sul calore specifico di soluzioni non elettrolitiche »(p. 283). - Il Magie trova che per lo più il calore molecolare di un corpo in soluzione è costante.

Il calore molecolare della glicerina mescolata con molta acqua sarebbe 54,1, mescolata con alcool etilico sarebbe molto minore; quello dell'acqua mescolata con molta anilina salebbe quasi il doppio del valore ohe si ottiene direttamence. Per esaminare questo complesso di cose l'A. propone lo studio di soluzioni di acqua e anilina con molta acqua, $\theta$ di glicerina e alcool con molta glicerina.

\title{
A. Amerto.
}

Wiedemann's Annalen. Vol. IXVI, Ni. 9 e 10, 1898.

Winkelmann A. Sulle correnti elettriche che sono prodotte dai raggi $X$ di Röntgen (pp. 1-28). - L'A. con metodo differente ha ritrovato in parte i risultati gia ottenuti dal Perrin secondo i quali $i$ metalli si caricano, sotto l'azione dei raggi $X$, fino ad un certo potenziale differente da un metallo all'altro; $i$ motalli, dal punto di vista di questi potenziali, si dispongono secondo la legge delle tensioni di Volta e si possono cosi produrre delle correnti

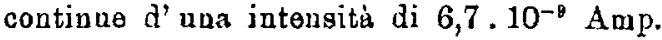

In seguito l'A. descrive minutamente un metodo col quale ha cercato di determinare la resistenza elettrioa di uno strato d'aria attraversato dai raggi di Röntgen, calcola il numero minimo di moleoule ionizzate contenute nell' unità di volume; trova 
che il rapporto fra il numero delle molecole ionizzate $\theta$ il numero totale $\dot{e}$, per l' aria, 4,6 $10^{-12}$.

NIETHAMmer F. Alcune ricerche sperimentali sull' isteresi magnetica (pp. 29-48). - L'A. ha studiato l' isteresi sopra uno stesso campione di ferro sottoponendolo successivamente ai tre modi seguenti di magnetizzazione: 1) Variazione lenta $\theta$ ben determinata della forza magnetizzante; 2) Magnetizzazione ciclica rapida, con correnti intermittenti o alternate; 3) Magnetizzazione ciclica per rotazione del campione in un campo magnetico costante.

I risultati più importanti trovati dall'A. sono:

1. La relazione di Steinmetz $A=\eta B^{1,6}$ che rappresenta il lavoro d'isteresi A per ciclo e per centimetro cubo in funzione dell' indazione massima raggiunta $B$, non $\dot{\theta}$ applicabile che fra limiti unolto vicini della forza magnetizzante, mentre il coefficiente $d^{\prime}$ 'isteresi $\eta$ varia notevolmente con $B$.

2. Per uno stesso valore di B la permeabilitá, come pure il lavoro d'isteresi, è più grande nel primo procedimento che nel secondo.

3. Colla rotazione in un campo magnetico, A cresce dapprima con $B$, poi, a partire da un certo valore di $B$ prossimo alla saturazione decresce.

4. Nel terzo procedimento, quando B è superiore a 4000 le scosse non hanno influenza notevole sopra l' isteresi.

STARKE H. Sopra la riflessione dei raggi catodici (pp. 49-60). - L'A. con un procedimento che egli descrive minutamente trova che i poteri riflettori dei vari tnetalli pei raggi catodici sono differentissini e proporzionali, almeno in generale, alla densità; cosi, se il platino ha un potere rappresentato da 21,6, l'alluminio ha un potere rappresentato da 2,6. Per una stessa intensitz dei raggi incidenti, le cariobe rillesse e assorbite sono complementari.

Wiadeanan E. Trasformazione dell' energia dei raggi catodici in energia di raggi luminosi (pp. 61-64).

Gertukr (von) J. R. Sulla differenza nella natura fisica dei raggi catodici e dei raggi di Röntgen (pp. 65-73). - L'A. mostra con alcune esperienze ohe i raggi $X$ sono degli uguagliatori di potenziale, come era già stato notato dal Perrin, mentre i raggi catodici sono delle correnti di elettricità negativa.

Walter B. Sulla natura dei raggi di Röntgen (pp. 74-82). Secondo l'A., i raggi di Röntgen sarebbero costituiti dalle particelle dei raggi catodici, ohe sarebbero riflesse e diffuse in tutti 
i sensi dall' anticatodo, dopo aver cedato a quest' ultimo la loro carica elettrica.

In quest' ipotesi si spiegano abbastanza facilmente le diffe. renze fra i due generi di raggi.

'Ooldenaar D. F. Osservazioni sul lavoro di L. Wiedemann sopra l' influenza reciprocs delle varie parti di un catodo (pp. 83-87).

Stgwart W. Sulla polverizzazione di fili di platino e di palladio, resi incandescenti dalk corrente (pp. 88-91). - L'A. trova che questa polverizzazione $\dot{\theta}$ quasi inseasibile o nulla nell'sdrogeno e nell' azoto; è invece considerevolo nell' aria e nell'ossigeno.

Wiedeburg O. Confronto fra gli irraggianenti calorifici dei metalli (pp. 92-110). - Lo scopo di questo Lavoro è il confronto dei poteri emissivi dei differenti metalli o leghe portate a $100^{\circ}$. Il Inetodo impiegato é quello del oubo di Leslie.

Il metallo che serve di termine di confronto è l'argento lu. cido. Gli altri metalli, ugualmento lucidi, che sono stati studiati sono il rame, l'oro, etc, o l'ottone, I'acciaio, Ia manganina, il reotano e la lega manganese-rame contenente il $30 \%$ di manganege.

L'A. trova che :

1. I differenti unetalli puri si dispongono, dal punto di vista dei poteri emissivi crescenti, nel medesimo ordine come dal punto di vista delle loro conducibilitá elettriche e termiche decrescenti.

2. Per lo leghe e por il nichel, dall' esser granile la rosi. stenze electrica non si puó concludere che sia grande il potere emissivo; l'osservazione precedente non si paò in questo caso applicare.

Sohncke L. Sulla variazione dei calori specifici colla temperatura (pp. 111-115).

ABT A. Confronto di alcune qualità di acciaio fra loro e col nickel e colla magnetite di Maravicza, dal punto di vista del loro magnetismo rimanente (pp. 116.120). - L' acciaio al tungsteno prende un magnetismo rimanente molto più grande degli acciai impiegati comunemente per le calamite permanenti. In quanto alla magnetite essa è migliore dell' acciaio ordinario e dell' acciajo di Bessemer, ma è inferiore agli acciai fusi raffinati e all' acciaio al tungstono.

Wesedonck K. Alcune osservazioni sul potere conduttore dei gas delle fiamme (pp. 121-135). - Molti fisici studiarono gia le 
circostanze nelle quali i gas perdono la loro elettrizzaziono o la proprieta di scaricare i corpi elettrizzati.

J. J. Thomson aveva osservato che l'aria sottoposta ai raggi di Röntgun sembra perdere ogai traccia di conducibilita quando è attraversata da goccie d'acqua o quando si fa filtrare attraverso ovatta o cotone di vetro. Ma esperienze posteriori di Lord Kelvin hanno mostrato che ò difficilissimo scaricar'e completamente il gas. La filtrazione attraverso l'ovatta è più efficace delle goccie d'acqua, ma neppur essa basta per ottenere una completa neutra. lizzazione.

L'A. ha studiato sotto questo punto di vista i gas usciti dalle fiamme ed ha osservato che la tillrazione attraverso l' ovatta toglie loro facilmente e completameute il potere conduttore. $\mathrm{Ma}$, avendo sottoposto questi gas all' azione delle goocie di un gran nu. mero di liquidi, ha osservato che nessuno di questi liquidi arrivava a produrre lo stesso effetto.

GoLnk (van) D. Sulla causa delle variazioni di resistenza scoperte da Branly (pp. 136-145). - L'A. discute varie ipotesi proposte per spiegare il fenomeno scoperto da Branly o descrive delle esperienze che confermano la teoria del contatto di Lodge.

DoRN E. Alcune osservazioni sui tubi a limatura (pp. 146 161). - L'A., avendo ricercato se $i$ raggi $X$ hanno un' jnfluenza sulla resistenza di foglio o di fili metallici, ha riconosciuto che la dimiuaziono di resistenza osservata non è dovata direttamente ai raggi $X$, ma alla scarica elettrica che li produce, e che la sede di questa variazione di resistenza risiede sulle superficie di contatto dei metalli.

In seguito l'A. ha studiato dei tubi a limatura costituiti da differenti metalli ed ha osservato ciò che avviene quando si fa il vuoto in questi tubi, mentre, per disseccarli, contemporaneamento vengono riscaldati, e ciù che avviene quando vi si lasciano rientrare differenti gas. Dalle sue esperienze risulta che il più grande ostacolo al passaggio della corrente è dovato agli stirati superficiali cattivi conduttori dei grani di limatura.

Neovrus 0 . Sulla probabile presenza nell' atmosfera di una sostanza fin qui incogniti (pp. 162-169). - Da ricerche spettro. scopiche eseguite sull' azoto estratto dall' aria, l'A. crede di poter dedurre che nell' aria esiste un corpo nuovo il quale vi si trova all' incirca belle stesse proporzioni dell'argon.

ZLNNECK J. Studio sulle lastre vibranti circolari (pp. 170176). - L'A. riprende o precisa le esperienze intraprese da Savart e studiate dipoi teoricamente da lord Rayleigh e Maltézos sopra le vibrazioni delle lastre circolari. 
Richarz H. o Krigar-Manzer O. Costante di gravitazione e densitì media della terra determinata con pesate (pp. 177-193). Gli A. descrivono una bilancia doppia formata da due serie di piatti posti 220 cun. gli uni al di sotto degli altri, colla quale poterono determinare il valore di $g$ e la densita media della terra. 'rovarono per quest' ultima il valore

$$
\Delta=(5,505+0,009) \underset{\mathrm{cm}^{i}}{\mathrm{gr}} .
$$

Volkmann P. Studi sulla tensione superficiale dell' acqua nei tubi capillari (pp. 194-206). - L'A. ha misurato la tensione superficiale dell' acqua nei tubi capillari di molte qualita di vetro, e specialmente dei vetri di Jena.

Scuulze F. A. Sopra un metodo per determinare la conducibilità dei corpi solidi (pp. 207-223). - Consideriamo una sbarra piuttosto lunga posta alla temperatura ambiente. Alla distanza di alcuni centimetri da una estremita nettiano un elemento termoelettrico in un foro praticato nella sbarra. All' istante $t=0$ la superficie terminale considerata è bagnata da una forte corrente d'acqua alla temperatura $\theta_{1}$, dopo $t_{1}$ secondi l'elemento termoelettrico indica una variazione di temperatura corrispondente allo spostamento di $n$ gradi sulla scala del galvanometro. Supponiamo che uno stesso spostamento di $n$ gradi si produca dopo $t_{2}$ secondi, quando la temperatura della corrente $d^{\prime}$ acqua $\dot{\theta}$ uguale a $\theta_{2}$; conoscendo $i$ valori $\theta_{1}, f_{2}, t_{1}, t_{2}$, si puó dedurre subito il coefficiente di conducibilità termica.

Hofrmanx W. Determinazione per l'aria del coefficiente di dilatarione a volume costante e della differenza fra il coeficiente di dilatazione a pressione costante e quello a volume costante (pp. 224-236). - L'A. impiegó due termometri ad aria di Jolly leggermente modificati. Fece esperienze a $0^{\circ}$ e a $100^{\circ}$, e rrovó pel coefficiente di dilatazione $\alpha_{p}$ a volume costante $\alpha_{p}=0,00366957 \theta$ per la differenza fra il coefficiente di dilatazione $x_{\gamma}$ a pressione costante (quella atmosferica) e il coefficiente $x_{p}$ il valore medio fra $0^{0}$ e $100^{\circ} \alpha_{r}-x_{p}=0,00000123$.

Berrx U. $S u l$ calore specifico di alcuni metalli alle basse temperature (pp. 237-244) - L'A. impiegò il metodo delle mescolanze. Raffreddo i metalli mettendoli sia nell' aria liquida $\left(-186^{\circ}\right)$, sia in una mescolanza di acido carbonico solido e alcool $\left(-80^{\circ}\right)$ e trovò che il calore specifico ra diminuendo colla temperatura e che questa diminuzione è tanto più grande quanto più è grande il ca. lore specifico. 
STARK J. Sulla distribuzione di corrente fra due elettrodi (pp. 245-268). - L'A. riassume i lavori anteriori e alcune sue ricerche, e mostra come i risultati si possono spiegare facilmente per mezzo di considerazioni teoriche che non si possono riassumere.

ERskine J. A. Sull' influenza reciproca di due circuiti; ap. plicazione alla determinazione delle custanti dielettriche dei liquidi (pp. 269-283).

\section{Pandolfi.}

P'hilosophical Hagazine. Vol. 48, Laglio e Agosto 1899.

WaIdNer C. W. e MaLlory F. Confronto fra il termometro a mercurio del Rowland con quello a platino di $C$. Griffith; fra il termometro a platino e uno Tonnelot campionato al Bureau International; e riduzione del valore dato da Rouland per l'equivalente meccanico del calore alla scala del termometro ad azoto di Parigi (pp. 1-45). - In questa nota sono descritte le operazioni eseguite per quei confronti $\theta$ per la ridazione indicata, e tutto ció con molti dettagli, che riescono di notevole interesse per chi si occupi di misure termometriche. Lo scopo di queste ricerche è stato di rendere le determinazioni dell'equivalente meccanico del calore fatte con metodi elettrici piu facilmente confrontabili con quelle fatte con metodi meccanici diretti.

MıLAR S. K. a Chatrock A. P. Sulla conduttività termica dell' acqua (pp. 46.64). - Il riscaldamento dell' acqua er a ottenuto mediante una spiralo metallica percorsa da una corrente elettrica, e mediante un metodo differenziale si evitavano gli errori pro. venienti dalle variazioni d'intensita della corrente. Il valore trovato per la conduttivita dell' acqua fu di 0,001433 C. G. S. a $20^{\circ}$.

KNoT' G. C. Riflessione e refrazione delle onde elastiche, con applicazione alla sismologia (pp. 64-97). - E uno stadio sulla propagazione delle onde sismiche, e della riflessione e refrazione che esse subiscono alla superficie di separazione fra due solidi, o fra un solido $\theta$ un fluido.

BeatTie J. C. Dispersione dell' elettricità dai corpi carichi, a temperature moderate (pp. 97-106). - L'A. ha studiato como varia la dispersione ricoprendo oon sostanze diverse alcune lastre metalliche isolate, contenute in una stufa la cui temperatura variava da quella della stanza fino a circa $300^{\circ}$. La differenza di potenziale fra la lastra e le pareti della stufa varió, in queste esperienze, da 50 a 240 volta. 